\title{
Prognostic benefit of surgical management in renal cell carcinoma patients with thrombus extending to the renal vein and inferior vena cava: 17-year experience at a single center
}

Shingo Hatakeyama', Takahiro Yoneyama', Itsuto Hamano', Hiromi Murasawa', Takuma Narita', Masaaki Oikawa', Kazuhisa Hagiwara', Daisuke Noro', Toshikazu Tanaka', Yoshimi Tanaka', Yasuhiro Hashimoto², Takuya Koie and Chikara Ohyama ${ }^{*}$

\begin{abstract}
Background: Management of renal cell carcinoma (RCC) with tumor thrombus extending to the renal vein and inferior vena cava (IVC) is challenging. The aim of this study was to evaluate the benefit of surgical management in such patients.

Methods: From February 1995 to February 2013, 520 patients were treated for RCC at Hirosaki University Hospital, Hirosaki, Japan. The RCC patients with tumor thrombus extending to the renal vein $(n=42)$ and IVC $(n=43)$ were included in this study. The records of these 85 patients were retrospectively reviewed to assess the relevant clinical and pathological variables and survival. Prognostic factors were identified by multivariate analysis. The benefit of surgical management was evaluated using propensity score matching to compare overall survival between patients who received surgical management and those who did not.

Results: RCC was confirmed by pathological examination of surgical or biopsy specimens in 74 of the 85 patients (87\%). Sixty-five patients (76\%) received surgical management (radical nephrectomy with thrombectomy). Distant metastasis was identified in 45 patients (53\%). The proportion of patients with tumor thrombus level 0 (renal vein only), I, II, III, and IV was 49\%, 13\%, 18\%, 14\%, and 5\%, respectively. The estimated 5-year overall survival rate was 70\% in patients with thrombus extending to the renal vein and $23 \%$ in patients with thrombus extending to the IVC. Multivariate analysis identified thrombus extending to the IVC, presence of distant metastasis, surgical management, serum albumin concentration, serum choline esterase concentration, neutrophil-lymphocyte ratio, and Carlson comorbidity index as independent prognostic factors. In propensity score-matched patients, overall survival was significantly longer in those who received surgical management than those who did not.
\end{abstract}

Conclusions: Surgical management may improve the prognosis of RCC patients with thrombus extending to the renal vein and IVC.

Keywords: Renal cell carcinoma, Radical nephrectomy with thrombectomy, Tumor thrombus, Prognostic factors

\footnotetext{
* Correspondence: coyama@cc.hirosaki-u.ac.jp

'Department of Urology, Hirosaki University Graduate School of Medicine,

5 Zaifu-chou, 036-8562 Hirosaki, Japan

Full list of author information is available at the end of the article
} 


\section{Background}

In renal cell carcinoma ( $\mathrm{RCC}$ ), thrombus extends to the inferior vena cava (IVC) in $4-15 \%$ of patients $[1,2]$. With recent advances in surgical techniques and instruments, radical nephrectomy with thrombectomy has been reported to improve the prognosis of RCC patients without distant metastasis [3]. However, surgery carries significant risks of perioperative morbidity and mortality [4]. Previous studies reported perioperative mortality rates of $0.1 \%$ for general surgery patients overall and $5-10 \%$ for patients undergoing radical nephrectomy with IVC thrombectomy [5-7]. Although several studies reported large series of RCC patients who underwent radical nephrectomy with thrombectomy [8-10], only a few studies compared outcomes between patients who underwent surgery and those who did not $[8,11]$. The role of nephrectomy with thrombectomy in patients with distant metastasis also remains unclear. In this study of RCC patients with renal vein and IVC thrombus, we compared outcomes between patients who received surgical management and those who did not.

\section{Methods}

\section{Patients and staging}

This study was performed in accordance with the ethical standards of the Declaration of Helsinki. The study protocol was approved by the institutional review board of Hirosaki University School of Medicine. A total of 520 RCC patients were treated in our clinic from February 1995 to February 2013. Of these, 42 patients had tumor thrombus extending to the renal vein (RV thrombus group) and 43 had tumor thrombus extending to the IVC (IVC thrombus group). The records of these 85 patients were retrospectively reviewed to assess the relevant clinical and pathological variables and survival.

All 85 patients underwent routine preoperative blood tests; brain, chest, and abdominal computed tomography (CT); abdominal magnetic resonance imaging; and/or bone scintigraphy. Gross extension of tumor thrombus into the venous system was detected by preoperative radiological examinations, including contrast-enhanced CT, magnetic resonance imaging, and/or vena cavography.

The level of tumor thrombus was determined according to the Mayo classification (Figure 1): level 0, thrombus extending to the renal vein only; level I, thrombus extending into the IVC to no more than $2 \mathrm{~cm}$ above the renal vein; level II, thrombus extending into the IVC to more than $2 \mathrm{~cm}$ above the renal vein but not to the hepatic vein; level III, thrombus extending into the IVC to above the hepatic vein but not to the diaphragm; and level IV, thrombus extending into the supradiaphragmatic IVC or right atrium [12]. Pathological diagnoses were determined according to the 2009 Union for International Cancer Control/American Joint Committee on Cancer

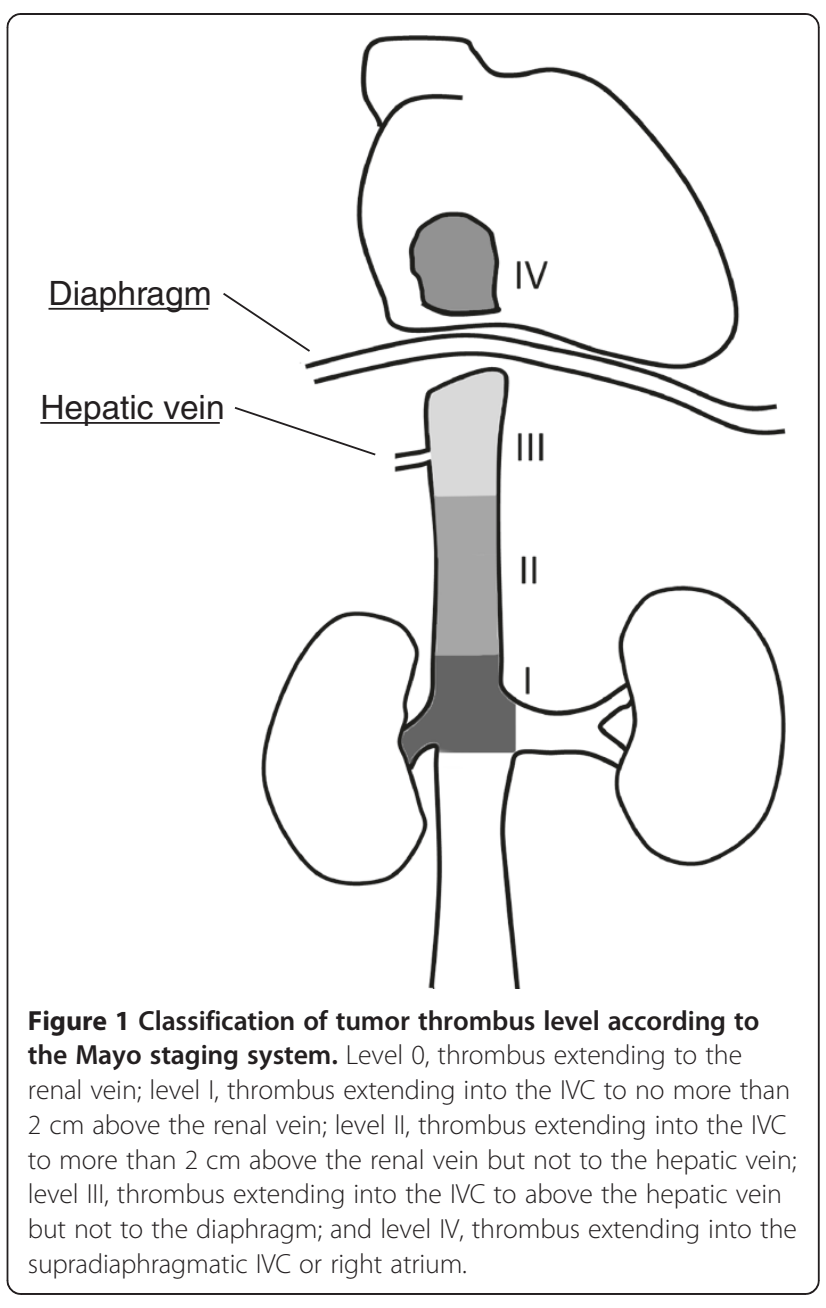

TNM system $[13,14]$. Overall patient condition was assessed using the Eastern Cooperative Oncology Group performance status (ECOG-PS) [15].

The basic treatment strategy for RCC with tumor thrombus was surgical extirpation of the tumor, with the aim of prolonging survival. Patients received nonsurgical management if they refused surgery or if they had worsening systemic comorbidities, ECOG-PS >3, extremely advanced metastatic disease that would be difficult to control, or severe complications.

\section{Surgical management}

All patients who received surgical management underwent radical nephrectomy, thrombectomy, and lymph node dissection. Surgery was performed via a flank or midline abdominal incision, depending on surgeon preference and the characteristics of the tumor and associated thrombus. In patients with supradiaphragmatic IVC thrombus, the liver was mobilized to expose the retrohepatic vena cava by incision of the falciform, triangular, and coronary ligaments, in cooperation with the Department of Gastroenterological Surgery. In patients with right atrium thrombus, sternotomy 
and extracorporeal circulation with cardiopulmonary bypass were performed in cooperation with the Department of Cardiothoracic Surgery.

\section{Follow-up schedule}

Patients were evaluated for postoperative recurrence and general condition by blood count, blood biochemistry analysis, and chest and abdominal CT every 3 months for the first year, and every 6 months thereafter. Chest CT was used instead of chest X-ray in consideration of the relative risks and benefits of these examinations. Brain CT was performed when any new metastatic lesions or neurological symptoms were observed. Patients did not receive routine postoperative adjuvant therapy, but additional treatment was given when new metastatic lesions were identified.

\section{Statistical analysis}

Patients in the RV thrombus group and IVC thrombus group were analyzed separately. To evaluate the benefit of surgical management, we compared overall survival between propensity score-matched patients (difference in score $\leq 0.03$ ) who received surgical management and those who did not. Propensity scores were calculated by logistic analysis using conventionally recognized risk factors for survival at the time of the initial visit, including age, sex, ECOG-PS, level of tumor thrombus, presence of distant metastasis, and Charlson comorbidity index.

Survival was calculated using the Kaplan-Meier method. The significance of differences between groups was evaluated using the log-rank test. Variables were compared between groups using the Student's $t$-test or Mann-Whitney $U$ test. Prognostic factors were identified by univariate and multivariate analyses using the Cox proportional hazards model, and hazard ratios (HRs) with 95\% confidence intervals were calculated. All statistical analyses were performed using the SPSS software package version 19.0 (SPSS, Chicago, IL, USA) and GraphPad Prism version 5.03 (GraphPad Software, San Diego, CA, USA). A value of $\mathrm{P}<0.05$ was considered statistically significant.

\section{Results}

Patient characteristics are shown in Table 1 and overall survival is shown in Figure 2. The diagnosis of RCC was confirmed by pathological examination of surgical or biopsy specimens in $74 / 85$ patients $(87 \%)$ and by imaging examination findings in 11 patients (13\%). Fortytwo patients $(49 \%)$ had thrombus extending to the renal vein and 43 (51\%) had thrombus extending to the IVC. In patients with IVC thrombus, the thrombus was classified as level I, II, III, and IV in 11 patients (13\%), 15 patients (18\%), 12 patients (14\%), and 5 patients (6\%), respectively. The prevalence of multiple organ metastases in surgical and non-surgical treatment group was $6 \%$ and $57 \%$ in renal vein, $14 \%$ and $50 \%$ in level I, 15\% and $100 \%$ in level II, 25\% and 50\% in level III, 0\% and 67\% in level IV, respectively.

Sixty-five patients $(76 \%)$ underwent radical nephrectomy with thrombectomy, and 20 did not receive surgical management. None of the patients who received surgical management underwent preoperative renal artery embolization. The median follow-up period was 26 months in patients who received surgical management and 5 months in patients who did not. Among the patients who did not receive surgical management, eight received immunotherapy or interferon- $\alpha 6,000,000$ IU three times/week, seven received molecular targeted therapy, one underwent tumor embolization, and four received best supportive care only. The reason for non-surgical management was multiple organ or unresectable metastasis in 14 patients (lung and lymph nodes, $\mathrm{n}=6$; lung and bone, $\mathrm{n}=2$; lung, $\mathrm{n}=2$; lung, bone, and brain, $\mathrm{n}=1$; lung and liver in a patient with duodenal invasion, $\mathrm{n}=1$; brain, $\mathrm{n}=1$; lymph nodes, $\mathrm{n}=1$ ), patient refusal in 4 patients, dementia in 1 patient, and ECOG-PS $>3$ in 1 patient. In the whole group of 85 patients, the estimated median overall survival time was 41 months and the estimated 5-year overall survival rate was $40 \%$ (Figure $3 \mathrm{~A}$, Table 2). At the time of this report, 43 patients (51\%) had died of their disease, including 24 (43\%) who received surgical management and $15(75 \%)$ who did not $(\mathrm{P}=0.003)$. In all patients who did not receive surgical management, the main cause of death was cachexia. In patients who received surgical management, the estimated median survival time was 60 months and the estimated 5-year overall survival rate was $54 \%$. In patients who did not receive surgical management, the estimated median survival time was 8.2 months and the estimated 5-year survival rate was $0 \%$ (Table 2). Distant metastasis was present at the time of diagnosis in 45 patients (53\%). In patients with distant metastasis at presentation, the median overall survival time was 11 months and the estimated 5-year survival rate was $21 \%$. In patients without distant metastasis at presentation, the estimated median survival time was 24 months and the estimated 5-year survival rate was $80 \%$ (Table 2). The independent prognostic factors identified by multivariate analysis using the Cox proportional hazards model were thrombus level, presence of distant metastasis, surgical management, serum albumin concentration, serum choline esterase concentration, neutrophil-lymphocyte ratio, and Charlson comorbidity index (Table 3 ).

\section{RCC patients with tumor thrombus extending to the renal vein}

Thirty-five of the 42 patients in the RV thrombus group received surgical management and 7 did not. None of these patients underwent metastasectomy, except for lymph node dissection. There were no significant differences between patients who received surgical management and those who 
Table 1 Patient characteristics

\begin{tabular}{|c|c|c|c|c|c|c|c|}
\hline & & & Renal vein & & & C thrombus & \\
\hline & All & $\begin{array}{c}\text { Surgery } \\
\text { performed }\end{array}$ & $\begin{array}{c}\text { Surgery not } \\
\text { performed }\end{array}$ & $P$ value & $\begin{array}{c}\text { Surgery } \\
\text { performed }\end{array}$ & $\begin{array}{c}\text { Surgery not } \\
\text { performed }\end{array}$ & $P$ value \\
\hline$n$ & 85 & 35 & 7 & & 30 & 13 & \\
\hline Age* $^{*}$ & $62 \pm 12$ & $63 \pm 10$ & $62 \pm 10$ & 0.790 & $59 \pm 14$ & $37 \pm 11$ & 0.078 \\
\hline Gender & & $24 / 11$ & $4 / 3$ & 0.558 & $17 / 13$ & $10 / 3$ & 0.021 \\
\hline ECOG-PS & $0.6 \pm 1.1$ & $0.3 \pm 0.7$ & $0.7 \pm 1.5$ & 0.484 & $0.5 \pm 1.0$ & $1.6 \pm 1.4$ & 0.021 \\
\hline Radical surgery & $65(76 \%)$ & 35 & 0 & & 30 & 0 & \\
\hline Perioperative morality within 1 month & & $0(0 \%)$ & & & $2(7 \%)$ & & \\
\hline Thrombus level & & & & & & & \\
\hline Renal vein & $42(49 \%)$ & 35 & 7 & & & & \\
\hline । & $11(13 \%)$ & & & & $7(23 \%)$ & $4(31 \%)$ & \\
\hline$\|$ & 15(18\%) & & & & $13(43 \%)$ & $2(15 \%)$ & \\
\hline III & $12(14 \%)$ & & & & $8(27 \%)$ & $4(31 \%)$ & 0.203 \\
\hline IV & $5(6 \%)$ & & & & $2(7 \%)$ & $3(23 \%)$ & \\
\hline Clinical TNM & & & & & & & \\
\hline cT3 & $78(92 \%)$ & $35(100 \%)$ & $6(86 \%)$ & & $30(100 \%)$ & $7(54 \%)$ & مค \\
\hline cT4 & $7(8 \%)$ & $0(0 \%)$ & $1(14 \%)$ & 0.024 & $0(0 \%)$ & $6(46 \%)$ & 0.000 \\
\hline $\mathrm{cN}+$ & $20(24 \%)$ & $1(3 \%)$ & $5(71 \%)$ & $<0.0001$ & $5(17 \%)$ & $9(69 \%)$ & 0.001 \\
\hline $\mathrm{CM}+$ & $45(53 \%)$ & $11(31 \%)$ & $6(86 \%)$ & 0.008 & $17(33 \%)$ & $11(85 \%)$ & 0.041 \\
\hline Multiple organ metastasis & 19(22\%) & $2(6 \%)$ & $4(57 \%)$ & 0.006 & $5(17 \%)$ & $8(62 \%)$ & 0.041 \\
\hline Thrombus level (I/II/III/IV) & & & & & $1 / 2 / 2 / 0$ & $2 / 2 / 2 / 2$ & \\
\hline Histology & & & & & & & \\
\hline Clear & $67(79 \%)$ & $31(89 \%)$ & $5(71 \%)$ & & $27(93 \%)$ & $4(31 \%)$ & \\
\hline Others & $7(8 \%)$ & $4(11 \%)$ & $0(0 \%)$ & 0.426 & $3(7 \%)$ & $0(0 \%)$ & 0.508 \\
\hline Unknown & $11(13 \%)$ & $0(0 \%)$ & $2(29 \%)$ & & $0(0 \%)$ & $9(96 \%)$ & \\
\hline Grade & & & & & & & \\
\hline $\mathrm{G} 1 / 2$ & $43(51 \%)$ & $25(71 \%)$ & $0(0 \%)$ & & $12(40 \%)$ & $4(31 \%)$ & \\
\hline G3 & $31(36 \%)$ & 10(29\%) & $5(71 \%)$ & 0.002 & $12(40 \%)$ & $4(31 \%)$ & 0.024 \\
\hline Unknown & $11(13 \%)$ & $0(0 \%)$ & $2(29 \%)$ & & $0(0 \%)$ & $9(69 \%)$ & \\
\hline Hemoglobin $(\mathrm{g} / \mathrm{dll})^{*}$ & $11.6 \pm 2.4$ & $12.0 \pm 2.2$ & $104 \pm 2.0$ & 0.081 & $11.4 \pm 2.3$ & $11.2 \pm 3.3$ & 0.752 \\
\hline Neutrophil-lymphocyte ratio* & $3.1 \pm 1.5$ & $2.8 \pm 1.2$ & $3.6 \pm 1.2$ & 0.156 & $3.1 \pm 1.9$ & $3.6 \pm 1.5$ & 0.402 \\
\hline Serum albumin $(g / d l)^{*}$ & $3.8 \pm 0.6$ & $3.9 \pm 0.6$ & $3.4 \pm 0.8$ & 0.122 & $3.9 \pm 0.6$ & $3.5 \pm 1.5$ & 0.080 \\
\hline Renal function $\left(\mathrm{ml} / \mathrm{min} . / 1.73 \mathrm{~m}^{2}\right)^{*}$ & $63 \pm 23$ & $71 \pm 20$ & $52 \pm 21$ & 0.063 & $60 \pm 23$ & $56 \pm 25$ & 0.605 \\
\hline Lactate dehydrogenase $(\mathrm{LDH}, \mid \mathrm{UU} / \mathrm{L})^{*}$ & $214 \pm 112$ & $185 \pm 45$ & $304 \pm 217$ & 0.198 & $199 \pm 80$ & $274 \pm 172$ & 0.154 \\
\hline Choline esterase $(\mathrm{U} / \mathrm{L})^{*}$ & $224 \pm 105$ & $248 \pm 98$ & $234 \pm 72$ & 0.665 & $215 \pm 130$ & $172 \pm 102$ & 0.252 \\
\hline Serum sodium $(\mathrm{mEq} / \mathrm{L})^{*}$ & $141 \pm 2.5$ & $142 \pm 2.3$ & $139 \pm 3.7$ & 0.111 & $142 \pm 2.2$ & $139 \pm 2.3$ & 0.005 \\
\hline Correlated calcium $(\mathrm{mg} / \mathrm{dL})^{*}$ & $9.6 \pm 0.8$ & $9.4 \pm 0.6$ & $9.9 \pm 0.8$ & 0.153 & $9.6 \pm 0.8$ & $10.1 \pm 0.8$ & 0.069 \\
\hline C-reactive protein (CRP, $\mathrm{mg} / \mathrm{dL})^{*}$ & $4.4 \pm 5.4$ & $4.1 \pm 6.1$ & $4.8 \pm 3.3$ & 0.648 & $3.7 \pm 6.0$ & $6.4 \pm 4.6$ & 0.125 \\
\hline Charlson comorbidity index* & $7.7 \pm 3.4$ & $6.6 \pm 3.1$ & $11.3 \pm 1.6$ & $<0.0001$ & $7.2 \pm 3.1$ & $10.3 \pm 3.0$ & 0.004 \\
\hline Molecular targeted agents & $18(21 \%)$ & $5(14 \%)$ & $4(57 \%)$ & 0.012 & $6(20 \%)$ & $3(23 \%)$ & 0.549 \\
\hline Deceased & $43(51 \%)$ & $8(23 \%)$ & $4(57 \%)$ & 0.067 & $20(67 \%)$ & $11(85 \%)$ & 0.228 \\
\hline
\end{tabular}




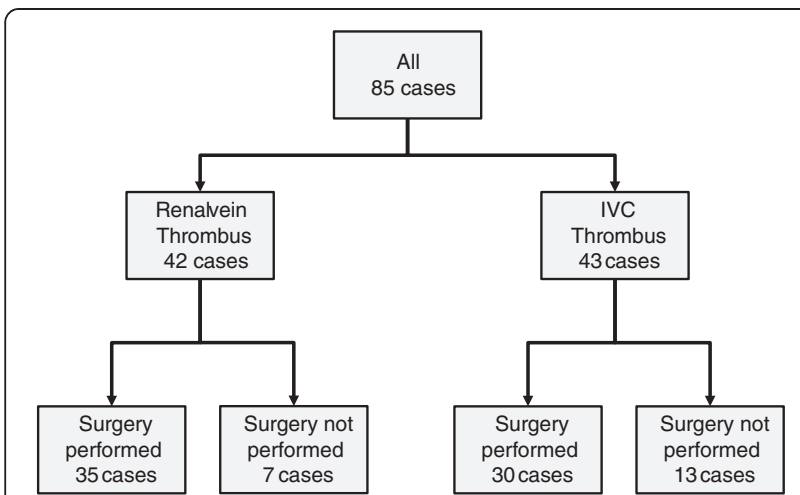

Figure 2 Management of enrolled patients. A total of 85 patients were enrolled in this study, including 42 in the RV group and 43 in the IVC group. Sixty-five patients underwent radical nephrectomy with thrombectomy and 20 did not undergo surgery.

did not in terms of age, sex, ECOG-PS, tumor histology, and blood biochemistry data. However, there were significant differences between these two groups in clinical $\mathrm{T}$ stage, lymph node involvement, presence of distant metastasis, tumor grade, Charlson comorbidity index, and administration of molecular targeted agents. In patients who received surgical management, the median blood loss was $744 \mathrm{~g}$ (range 10-3,221 g). Five patients with blood loss $>1,500$ g received blood transfusions. Two patients (6\%) developed perioperative complications of Clavien grade $\geq$ III, and there were no deaths within 1 month of surgery. No patients required cardiopulmonary bypass during surgery. In the whole RV thrombus group, the estimated median survival time was "undefined", and the estimated 5 -year survival rate was $70 \%$. In patients who received surgical management, the estimated median survival time was "undefined" and the estimated 5-year survival rate was $78 \%$. In patients who did not receive surgical management, the estimated median survival time was 4.9 months and the estimated 5-year survival rate was $0 \%$ (Figure 3B, Table 2). In patients with distant metastasis at presentation, the estimated median survival time was 26 months and the estimated 5-year survival rate was $42 \%$. In patients without distant metastasis at presentation, the estimated median survival time was "undefined" and the estimated 5-year survival rate was 90\% (Figure 3C, Table 2). The independent prognostic factors identified by multivariate analysis using the Cox proportional hazards model in the RV thrombus group were presence of distant

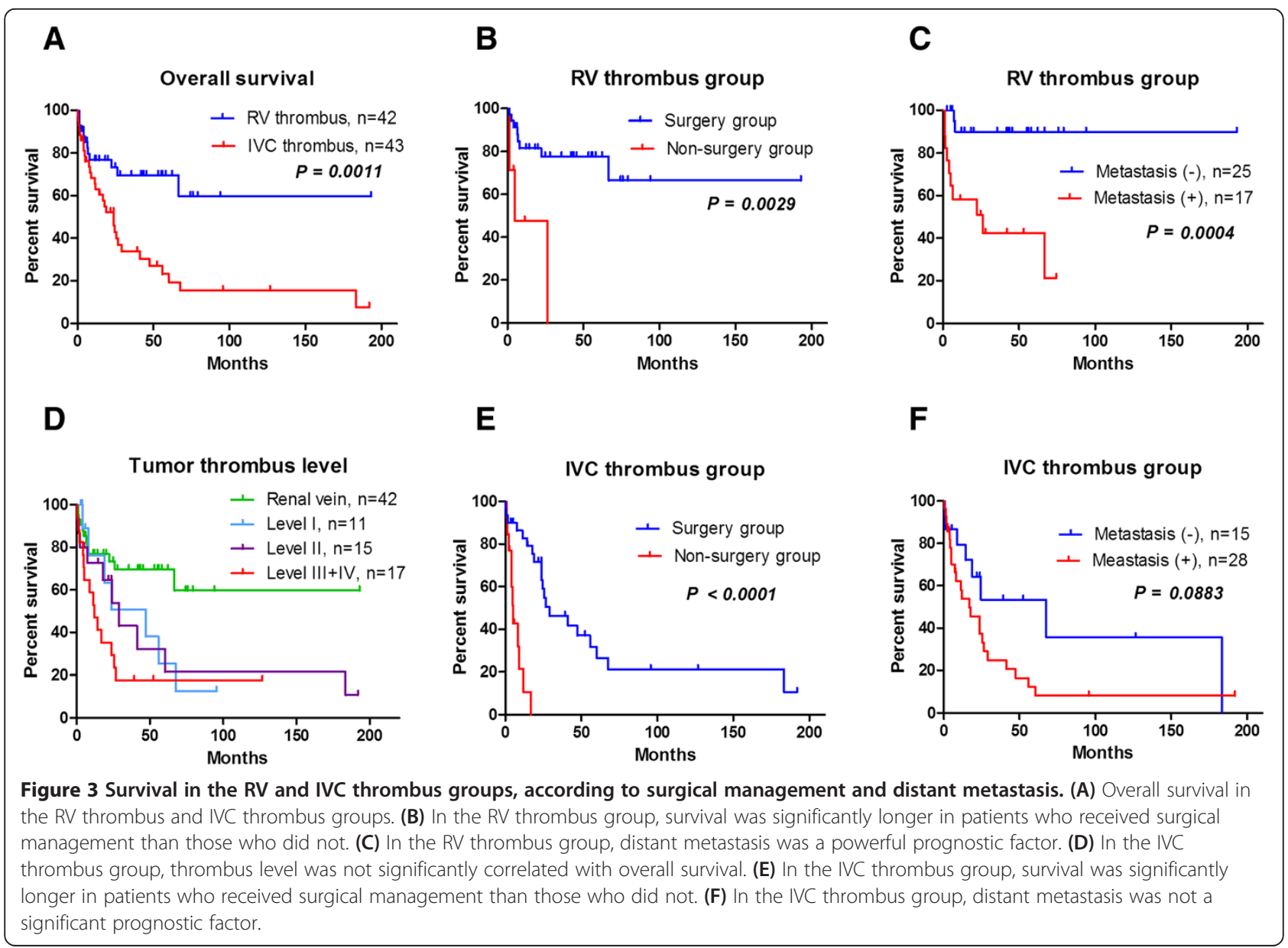


Table 2 Estimated median overall survival times and 5-year overall survival rates

\begin{tabular}{|c|c|c|c|c|c|}
\hline & All patients & Surgery group & Non-surgery group & Without metastasis & With metastasis \\
\hline Median survival (M) & 41 & 60 & 8.2 & 24 & 11 \\
\hline \multirow[t]{2}{*}{ 5-year survival (\%) } & 40 & 54 & 0 & 80 & 21 \\
\hline & RV thrombus & & & & \\
\hline Median survival (M) & Undefined & Undefined & 49 & Undefined & 26 \\
\hline \multirow[t]{2}{*}{ 5-year survival (\%) } & 70 & 78 & 0 & 90 & 42 \\
\hline & IVC thrombus & & & & \\
\hline Median survival (M) & 24 & 29 & 5.1 & 68 & 17 \\
\hline 5-year survival (\%) & 23 & 32 & 0 & 54 & 12 \\
\hline
\end{tabular}

metastasis and hemoglobin concentration. Surgical management was not an independent prognostic factor in this group (Table 3).

RCC patients with tumor thrombus extending to the IVC Thirty of the 43 patients in the IVC thrombus group received surgical management and 13 did not. Ten of these patients underwent metastasectomy (adrenal, $\mathrm{n}=1$; bone, $\mathrm{n}=1$; lymph nodes, $\mathrm{n}=2$; liver, $\mathrm{n}=1$; liver, $\mathrm{n}=2$; IVC, $n=4$ ) at the time of radical nephrectomy with thrombectomy. Four of these patients had positive surgical tumor margins, and these four all died within 3 years of surgery (median overall survival time: 26 months). There were significant differences between patients who received surgical management and those who did not in ECOG-PS, clinical $\mathrm{T}$ stage, lymph node involvement, tumor grade, serum sodium concentration, and Charlson comorbidity index. Other factors, including presence of distant metastasis, did not differ significantly between these two groups. In patients with thrombus level I, II, and III/IV who received surgical management, the median blood loss was $620 \mathrm{~g}$ (range 80-953 g), 1,255 g (range 810-5,100 g), and 3,397 g (range 2,454-13,800 g), respectively. Eighteen patients underwent blood transfusion because of blood loss $>1,000 \mathrm{~g}$ or hypotension. Cardiopulmonary bypass was performed in three patients. Eight patients (19\%) developed perioperative complications of Clavien grade $\geq$ III and two patients $(6.7 \%)$ died within 1 month of surgery. There was no correlation between the level of IVC tumor thrombus and overall survival (Figure 3D). In the whole IVC thrombus group, the estimated median survival time was 24 months and the estimated 5-year survival rate was $23 \%$. In patients who received surgical management, the estimated median survival time was 29 months and the estimated 5-year survival rate was $32 \%$. In patients who did not receive surgical management, the estimated median survival time was 5.1 months and the estimated 5-year survival rate was $0 \%$ (Figure 3E, Table 2). In patients with distant metastasis at presentation, the estimated median survival time was 17 months and the estimated 5-year survival rate was $12 \%$. In patients without distant metastasis at presentation, the estimated median survival time was "undefined" and the estimated 5-year survival rate was 90\% (Figure 3F, Table 2). The independent prognostic factors identified by multivariate analysis using the Cox proportional hazards model in the IVC thrombus group were surgical management, serum albumin concentration, adjusted serum calcium concentration, and neutrophil-lymphocyte ratio (Table 3 ).

\section{Propensity score-matched analysis}

Propensity score-matched analysis was used to compare overall survival between patients who received surgical management and those who did not. Conventionally recognized risk factors for survival including age, sex, ECOG-PS, level of tumor thrombus, presence of distant metastasis, and Charlson comorbidity index were controlled for using logistic analysis. Thirty pair-matched patients (15 patients who received surgical management and 15 who did not) were selected for analysis. The characteristics of these 30 patients are shown in Table 4. The only significant difference between patients who received surgical management and those who did not, including both matched and unmatched variables, was in serum sodium concentration.

Among propensity score-matched patients, the overall survival time was significantly longer in those who received surgical management than those who did not. In patients who received surgical management, the estimated median survival time was 29 months and the estimated 5-year survival rate was $20 \%$. In patients who did not receive surgical management, the estimated median survival time was 7 months and the estimated 5-year survival rate was $0 \%$ (Figure 4 ).

\section{Discussion}

Aggressive surgical management with a hope of cure is currently the standard treatment for RCC patients with tumor thrombus extending to the RV and IVC, even though such surgery is challenging. In this study, the perioperative mortality rate in patients with IVC thrombus was $6.7 \%$, which is significantly higher than the overall 
Table 3 Univariate and multivariate analyses for overall survival

\begin{tabular}{|c|c|c|c|c|c|c|c|c|c|c|c|c|c|c|c|c|c|c|}
\hline \multirow[b]{3}{*}{ Independent factors } & \multicolumn{6}{|c|}{ All } & \multicolumn{6}{|c|}{ RV thrombus group } & \multicolumn{6}{|c|}{ IVC thrombus group } \\
\hline & \multicolumn{3}{|c|}{ Univariate } & \multicolumn{3}{|c|}{ Multivariate } & \multicolumn{3}{|c|}{ Univariate } & \multicolumn{3}{|c|}{ Multivariate } & \multicolumn{3}{|c|}{ Univariate } & \multicolumn{3}{|c|}{ Multivariate } \\
\hline & $P$ value & $\mathrm{HR}$ & $95.0 \% \mathrm{Cl}$ & $P$ value & $\mathrm{HR}$ & $95.0 \% \mathrm{Cl}$ & $P$ value & $\mathrm{HR}$ & $95.0 \% \mathrm{Cl}$ & $P$ value & $\mathrm{HR}$ & $95.0 \% \mathrm{Cl}$ & $P$ value & $\mathrm{HR}$ & $95.0 \% \mathrm{Cl}$ & $P$ value & $\mathrm{HR}$ & $95.0 \% \mathrm{Cl}$ \\
\hline Age & 0.875 & 0.997 & $0.972-1.024$ & & & & 0.645 & 1.013 & $0.958-1.072$ & & & & 0.877 & 1.002 & $0.974-1.032$ & & & \\
\hline ECOG-Performance status & 0.002 & 1.433 & $1.151-1.869$ & & & & 0.045 & 1.776 & $1.012-3.115$ & & & & 0.063 & 1.297 & $0.986-1.704$ & & & \\
\hline Gender (ref, female) & 0.073 & 0.576 & $1.315-1.053$ & & & & 0.018 & 0.234 & $0.070-0.776$ & & & & 0.086 & 0.860 & $0.414-1.787$ & & & \\
\hline $\begin{array}{l}\text { Thrombus level } \\
\text { (ref, renal vein) }\end{array}$ & 0.000 & 1.472 & $1.185-1.830$ & 0.034 & 1.275 & 1.0181 .599 & & & & & & & 0.179 & 1.315 & $0.882-1.960$ & & & \\
\hline $\begin{array}{l}\text { Distant metastasis } \\
\text { (ref, without metastasis) }\end{array}$ & 0.000 & 4.115 & $2.020-8.384$ & 0.019 & 2.548 & $1.168-5.560$ & 0.003 & 9.673 & $2.113-44.27$ & 0.000 & 34.01 & $5.368-215.6$ & 0.094 & 1.996 & $0.888-4.4483$ & & & \\
\hline $\begin{array}{l}\text { Underwent surgery } \\
\text { (ref, not performed) }\end{array}$ & 0.000 & 0.144 & $0.069-0.303$ & 0.000 & 0.239 & $0.108-0.532$ & 0.008 & 0.180 & $0.051-0.636$ & & & & 0.000 & 0.105 & $0.036-0.305$ & 0.000 & 0.061 & $0.016-0.230$ \\
\hline Hemoglobin (g/dL) & 0.002 & 0.807 & $0.704-0.926$ & & & & 0.005 & 0.645 & $0.477-0.873$ & 0.002 & 0.500 & $0.323-0.775$ & 0.056 & 0.863 & $0.742-1.004$ & & & \\
\hline Serum albumin $(\mathrm{g} / \mathrm{dL})$ & 0.003 & 0.487 & $0.306-0.778$ & 0.026 & 0.849 & $0.736-0.980$ & 0.124 & 0.537 & $0.244-1.186$ & & & & 0.004 & 0.408 & $0.221-0.756$ & 0.002 & 0.250 & $0.104-0.600$ \\
\hline eGFR (ml/min./1.73 m²) & 0.190 & 0.989 & $0.974-1.005$ & & & & 0.470 & 0.989 & $0.961-1.019$ & & & & 0.494 & 0.999 & $0.995-1.002$ & & & \\
\hline Choline esterase (U/L) & 0.052 & 0.996 & $0.994-1.000$ & 0.023 & 0.995 & $0.992-0.999$ & 0.156 & 0.996 & $0.991-1.002$ & & & & 0.002 & 1.407 & $1.137-1.740$ & 0.053 & 1.282 & $0.996-1.648$ \\
\hline $\begin{array}{l}\text { Neutrophil-lymphocyte } \\
\text { ratio }\end{array}$ & 0.007 & 1.297 & $1.072-1.571$ & 0.021 & 1.250 & $1.034-1.513$ & 0.387 & 1.204 & $0.791-1.835$ & & & & 0.002 & 1.407 & $1.137-1.740$ & 0.053 & 1.282 & $0.993-1.648$ \\
\hline Serum sodium (mEq/L) & 0.001 & 0.814 & $0.721-0.921$ & & & & 0.006 & 0.774 & $0.644-0.931$ & & & & 0.090 & 0.862 & $0.723-1.023$ & & & \\
\hline $\begin{array}{l}\text { Correlated calcium } \\
\text { (mg/dL) }\end{array}$ & 0.322 & 1.143 & $0.877-1.491$ & & & & 0.109 & 1.982 & $0.858-4.579$ & & & & 0.439 & 0.854 & $0.572-1.274$ & 0.038 & 0.598 & $0.368-0.973$ \\
\hline $\begin{array}{l}\text { Lactate dehydrogenase } \\
\qquad(\mathrm{LDH}, \mathrm{IU} / \mathrm{L})\end{array}$ & 0.000 & 1.004 & $1.002-1.006$ & & & & 0.071 & 1.004 & $1.001-1.008$ & & & & 0.019 & 1.003 & $1.001-1.006$ & & & \\
\hline $\begin{array}{l}\text { C-reactive protein } \\
\text { (CRP, mg/dL) }\end{array}$ & 0.044 & 1.046 & $1.001-1.093$ & & & & 0.032 & 1.083 & $1.007-1.165$ & & & & 0.299 & 1.029 & $0.975-1.0865$ & & & \\
\hline $\begin{array}{l}\text { Charlson comorbidity } \\
\text { index }\end{array}$ & 0.000 & 1.258 & $1.130-1.401$ & 0.006 & 1.196 & $1.053-1.360$ & 0.002 & 1.442 & $1.144-1.818$ & & & & 0.022 & 1.152 & $1.020-1.3007$ & & & \\
\hline $\begin{array}{l}\text { Molecular targeted } \\
\text { agents (ref, not used) }\end{array}$ & 0.704 & 1.148 & $0.561-2.351$ & & & & 0.661 & 0.711 & $0.155-3.275$ & & & & 0.466 & 1.362 & $0.593-3.124$ & & & \\
\hline
\end{tabular}


Table 4 Characteristics of the patients included in the propensity score-matched analysis

\begin{tabular}{|c|c|c|c|}
\hline Propensity score matched patients & Surgery performed & Surgery not performed & $P$ value \\
\hline \multicolumn{4}{|c|}{$<$ Matched parameters $>$} \\
\hline$n$ & 15 & 15 & \\
\hline Age $^{*}$ & $63 \pm 11$ & $63 \pm 11$ & 0.923 \\
\hline Gender (male/female) & $9 / 6$ & $9 / 6$ & 1.000 \\
\hline ECOG-PS* & $0.7 \pm 1.2$ & $0.9 \pm 1.1$ & 0.758 \\
\hline Presence of distant metastasis $(\mathrm{M}+)$ & $13(87 \%)$ & $12(80 \%)$ & 0.624 \\
\hline Thrombus level & & & 0.624 \\
\hline Renal vein & 7 & 6 & \\
\hline । & 1 & 2 & \\
\hline$\|$ & 4 & 2 & \\
\hline III & 2 & 3 & \\
\hline IV & 1 & 2 & \\
\hline \multicolumn{4}{|c|}{$<$ Not matched parameters $>$} \\
\hline \multicolumn{4}{|l|}{ Clinical TN } \\
\hline cT3 & $15(100 \%)$ & $12(80 \%)$ & \multirow{2}{*}{0.068} \\
\hline cT4 & $0(0 \%)$ & $3(20 \%)$ & \\
\hline $\mathrm{CN}+$ & $5(33 \%)$ & $10(66 \%)$ & 0.068 \\
\hline Hemoglobin $(\mathrm{g} / \mathrm{dL})^{*}$ & $11 \pm 2.4$ & $11 \pm 3.2$ & 0.857 \\
\hline Neutrophil-lymphocyte ratio* & $3.2 \pm 1.5$ & $3.5 \pm 1.2$ & 0.636 \\
\hline Serum albumin $(\mathrm{g} / \mathrm{dL})^{*}$ & $3.8 \pm 0.5$ & $3.6 \pm 0.6$ & 0.331 \\
\hline Renal function $\left(\mathrm{ml} / \mathrm{min} . / 1.73 \mathrm{~m}^{2}\right)^{*}$ & $60 \pm 16$ & $58 \pm 20$ & 0.756 \\
\hline Lactate dehydrogenase (LDH, IU/L)* & $200 \pm 57$ & $279 \pm 201$ & 0.160 \\
\hline Choline esterase $(U / L)^{*}$ & $240 \pm 110$ & $188 \pm 107$ & 0.213 \\
\hline Serum sodium (mEq/L)* & $141 \pm 1.9$ & $139 \pm 3.0$ & 0.028 \\
\hline Correlated Calcium (mg/dL)* & $9.9 \pm 1.0$ & $9.9 \pm 0.7$ & 0.977 \\
\hline C-reactive protein $(\mathrm{CRP}, \mathrm{mg} / \mathrm{dL})^{*}$ & $6.0 \pm 7.3$ & $5.3 \pm 4.6$ & 0.787 \\
\hline Charlson comorbidity index* & $10 \pm 1.5$ & $10 \pm 2.5$ & 0.864 \\
\hline Molecular targeted argents & $6(40 \%)$ & $6(40 \%)$ & 1.000 \\
\hline Deceased & $10(67 \%)$ & $11(73 \%)$ & 0.690 \\
\hline
\end{tabular}

*(Mean \pm SD).

perioperative mortality rate of $<0.1 \%$ in patients who undergo urological surgery at our institute. Although many studies reported benefits associated with surgical management in RCC patients with tumor thrombus extending to the RV and IVC [10,16-18], only a few studies compared outcomes between patients who underwent surgery and those who did not $[8,11]$. No randomized studies assessing the benefits of surgical management in RCC patients with a poor prognosis have been reported, because the decisions regarding surgical management of these patients depend on factors such as performance status, severe comorbidities, and the presence of metastasis. The largest reported series of RCC patients with renal vein and IVC thrombus, which included 1,122 patients with a median follow-up period of 24.7 months, found a median overall survival time of 33.8 months [19]. However, the role of surgical management in that series is unclear because outcomes were not compared between RCC patients with tumor thrombus who underwent surgery and those who did not. In this study, we used propensity score-matched analysis to retrospectively compare outcomes between RCC patients with renal vein and IVC thrombus who received surgical management and those who did not.

In all 85 patients studied, the following factors were identified as independent predictors of overall survival: thrombus level, presence of distant metastasis, surgical management, serum albumin concentration, serum choline esterase concentration, neutrophil-lymphocyte ratio, and Charlson comorbidity index.

This study found that patients who underwent radical nephrectomy with thrombectomy survived longer than those who received non-surgical management. However, 


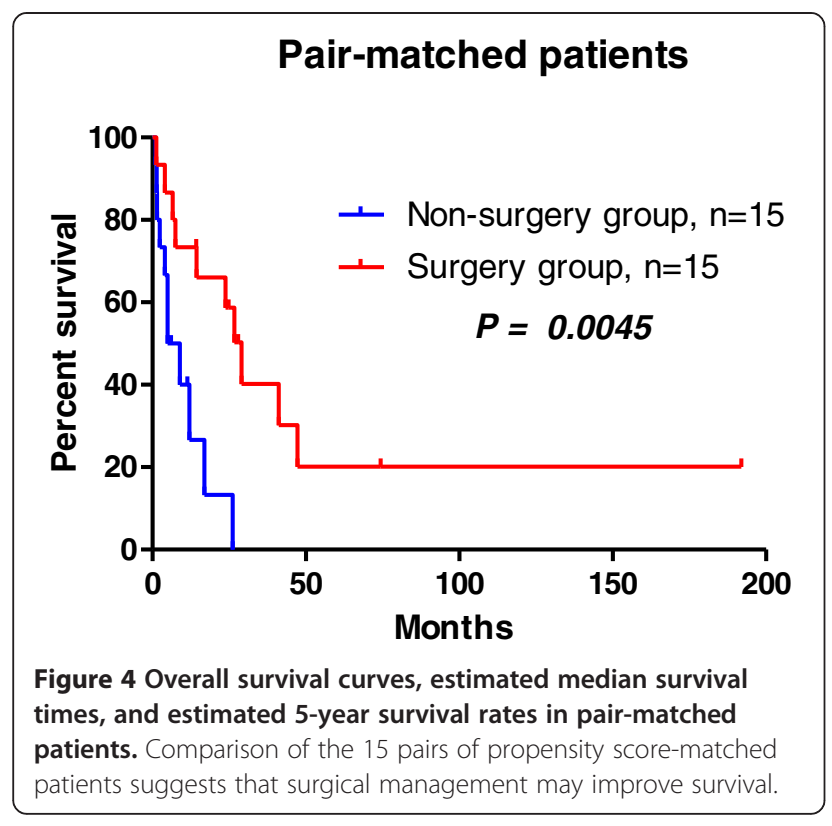

the significance of this observation is limited by selection bias and the significant differences in variables between patients who received surgical management and those who did not. Patients who did not receive surgical management had a higher ECOG-PS and greater tumor burden, including unresectable disease and metastasis. The median survival times in patients who received surgical management and those who did not are comparable with those previously reported $[8,11,18]$.

In the RV thrombus group, most patients (83\%) received surgical management. Patients with thrombus that does not extend beyond the renal vein are more likely to have organ-confined disease and to be surgical candidates than patients with IVC thrombus. There were no significant differences between patients with thrombus extending to the renal vein who received surgical management and those who did not in terms of age and ECOG-PS, but there were significant differences between these two groups in organ-confined disease, presence of distant metastasis, tumor grade, Charlson comorbidity index, and administration of targeting agents. These findings suggest that RCC patients with more advanced disease were less likely to receive surgical management. The estimated median survival time was significantly longer in patients who received surgical management than in those who did not ("undefined" vs. 4.9 months). The estimated median survival times in this study are comparable with those previously reported $[8,10]$. Multivariate analysis of the RV thrombus group identified presence of distant metastasis and hemoglobin concentration as independent prognostic factors for overall survival. The estimated 5 -year survival rate was $90 \%$ in patients with distant metastasis and $42 \%$ in patients without distant metastasis.
In the IVC thrombus group, $70 \%$ of patients received surgical management. There were no significant differences between patients who received surgical management and those who did not in terms of age, thrombus level, or presence of distant metastasis, but there were significant differences between these two groups in ECOG-PS, clinical $\mathrm{T}$ stage, clinical $\mathrm{N}$ stage, tumor grade, serum sodium concentration, and Charlson comorbidity index. These findings suggest that patients with advanced RCC and poor general status were more likely to receive nonsurgical management. As in the RV thrombus group, the estimated median survival time was significantly longer in patients who received surgical management than those who did not ( 29 vs. 5.1 months, $\mathrm{P}<0.0001)$. The estimated median survival times in this study are comparable with those previously reported $[8,10,11,18]$. Multivariate analysis of the IVC thrombus group identified surgical management, serum albumin concentration, neutrophil-lymphocyte ratio, and adjusted serum calcium concentration as independent prognostic factors for overall survival; but not thrombus level or presence of distant metastasis. The relationship between the level of IVC thrombus and prognosis is currently unclear [10,19-21]. Kim et al. reported that the level of tumor thrombus was not a predictor of cancer-specific survival on multivariate analysis ( $\mathrm{HR} 0.95, \mathrm{P}=0.872$ ) [20]. In contrast, Martinez-Salamanca et al. reported that tumor thrombus extending above the diaphragm ( $\mathrm{HR} 2.10, \mathrm{P}=0.00$ ), tumor diameter $>7 \mathrm{~cm}$, Furman grade, fat invasion, lymph node metastasis, and presence of distant metastasis were independent predictors of cancer-specific survival [19]. In this study, thrombus level of IVC was not identified as a significant predictor of overall survival. There was also no significant difference in overall survival between patients with suprahepatic IVC thrombus and those with infrahepatic IVC thrombus only (data not shown). This lack of association between thrombus level and survival may be due to the relatively small sample size.

The presence of distant metastasis has been reported to be a very powerful prognostic factor in RCC patients with tumor thrombus [10]. Lambert et al. studied RCC patients with venous tumor thrombus, and found a 5-year cancer-specific survival rate of $10.0 \%$ in patients with distant metastasis and $60.3 \%$ in those without distant metastasis [22]. Although the presence of distant metastasis was not identified as an independent prognostic factor in this study, the estimated 5-year overall survival rate was significantly lower in patients with distant metastasis than those without ( $12 \%$ vs. $54 \%, \mathrm{P}=0.0883)$.

We used propensity score-matched analysis to control for patient characteristics when comparing overall survival between patients who received surgical management and those who did not. The variables used for the logistic analysis were conventionally recognized risk factors for 
survival, including age, sex, ECOG-PS, level of tumor thrombus, presence of distant metastasis, and Charlson comorbidity index. Table 4 shows the matched and unmatched variables in patients who received surgical management and those who did not. Comparison of the 15 pairs of propensity score-matched patients suggests that surgical management may improve survival. The estimated median survival time was four times longer in patients who received surgical management than those who did not $(\mathrm{P}=0.0045)$.

The findings of this study and of previous studies $[3,8-10,17,18,23]$ suggest that the upper limit of the 5-year survival rate in RCC patients with venous thrombus who receive surgical management may be $50-60 \%$. Because these patients have a high rate of metastasis at presentation, optimal systemic therapy, such as neoadjuvant therapy using molecular-targeting agents, is important. The first report of neoadjuvant sunitinib therapy in an RCC patient with IVC thrombus described a significant down-staging of the thrombus and reduction of the extent of surgery required [24]. Subsequent studies reported significant reduction in tumor thrombus after preoperative sunitinib [25] or sorafenib therapy [16]. These data suggest that neoadjuvant therapy can downsize thrombus with minimal invasiveness, thereby increasing surgical resectability. However, some studies did not report good results after neoadjuvant therapy [23,26]. In 2011, Cost et al. reported that targeted therapy resulted in only a $12 \%$ cytoreductive effect and a high rate of progression. The usefulness of neoadjuvant targeted therapy therefore remains unclear.

This study has several limitations, including the small sample size and the inclusion of only patients treated at a single institution. These factors make it difficult to control for selection bias and patient characteristics. However, an advantage of this study is the use of propensity score matching between patients who received surgical management and those who did not. The results show an overall survival benefit for patients who received surgical management. Randomized trials including larger numbers of patients from multiple institutions are necessary to clarify the benefits of surgical management in patients with RCC with venous thrombus.

\section{Conclusions}

Surgical management may improve the prognosis of RCC patients with thrombus extending to the renal vein and IVC.

\section{Abbreviations}

CT: Computed tomography; ECOG-PS: Eastern cooperative oncology group performance status; IVC: Inferior vena cava; RCC: Renal cell carcinoma; RV: Renal vein

\section{Competing interests}

The authors declare that they have no competing interests.

\section{Authors' contributions}

$\mathrm{SH}$ drafted the manuscript. TK participated in drafting the manuscript. TY, $I H$, $\mathrm{HM}, \mathrm{TN}, \mathrm{MO}, \mathrm{KH}, \mathrm{DN}, \mathrm{TT}, \mathrm{YT}$, and $\mathrm{YH}$ performed the clinical follow-up and collected data. CO was responsible for the concept and design of the study, the interpretation of data, and critical revision of the manuscript. All authors read and approved the final manuscript.

\section{Authors' information}

SH: lecturer; TY: lecturer; $1 \mathrm{H}$ : resident in training; HM: postgraduate student; $\mathrm{TN}$ : postgraduate student; $\mathrm{MO}$ : postgraduate student; $\mathrm{KH}$ : postgraduate student; DN: postgraduate student; T: postgraduate student; YT: postgraduate student; $\mathrm{YH}$ : associate professor; TK: associate professor; CO: professor and chairman, Department of Urology, Hirosaki Graduate School of Medicine.

\section{Acknowledgments}

This work was supported by a Grant-in-Aid for Scientific Research (No. 23791737) from the Japan Society for the Promotion of Science.

\section{Author details}

'Department of Urology, Hirosaki University Graduate School of Medicine, 5 Zaifu-chou, 036-8562 Hirosaki, Japan. ${ }^{2}$ Department of Advanced Transplant and Regenerative Medicine, Hirosaki University Graduate School of Medicine, Hirosaki, Japan.

Received: 16 July 2013 Accepted: 11 October 2013

Published: 14 October 2013

\section{References}

1. Ljungberg B, Stenling R, Osterdahl B, Farrelly E, Aberg T, Roos G: Vein invasion in renal cell carcinoma: impact on metastatic behavior and survival. J Urol 1995, 154(5):1681-1684.

2. O'Donohoe MK, Flanagan F, Fitzpatrick JM, Smith JM: Surgical approach to inferior vena caval extension of renal carcinoma. Brit J Urol 1987, 60(6):492-496

3. Vander Eeckt K, Joniau S, Van Poppel H: Open surgery for localized RCC. Sci World J 2007, 7:742-752.

4. Skinner DG, Pritchett TR, Lieskovsky G, Boyd SD, Stiles QR: Vena caval involvement by renal cell carcinoma. Surgical resection provides meaningful long-term survival. Ann Surg 1989, 210(3):387-392. discussion 392-384.

5. Bainbridge D, Martin J, Arango M, Cheng D: Perioperative and anaesthetic-related mortality in developed and developing countries: a systematic review and meta-analysis. Lancet 2012, 380(9847):1075-1081

6. Blute $\mathrm{ML}$, Leibovich $\mathrm{BC}$, Lohse $\mathrm{CM}$, Cheville JC, Zincke $\mathrm{H}$ : The Mayo Clinic experience with surgical management, complications and outcome for patients with renal cell carcinoma and venous tumour thrombus. BJU Int 2004, 94(1):33-41.

7. Al Otaibi M, Abou Youssif T, Alkhaldi A, Sircar K, Kassouf W, Aprikian A Mulder D, Tanguay S: Renal cell carcinoma with inferior vena caval extention: impact of tumour extent on surgical outcome. BJU Int 2009, 104(10):1467-1470.

8. Zisman A, Wieder JA, Pantuck AJ, Chao DH, Dorey F, Said JW, Gitlitz BJ, deKernion JB, Figlin RA, Belldegrun AS: Renal cell carcinoma with tumor thrombus extension: biology, role of nephrectomy and response to immunotherapy. J Urol 2003, 169(3):909-916.

9. Tanaka M, Fujimoto K, Okajima E, Tanaka N, Yoshida K, Hirao Y: Prognostic factors of renal cell carcinoma with extension into inferior vena cava. Int J Urol 2008, 15(5):394-398.

10. Miyake H, Terakawa T, Furukawa J, Muramaki M, Fujisawa M: Prognostic significance of tumor extension into venous system in patients undergoing surgical treatment for renal cell carcinoma with venous tumor thrombus. Eur J Surg Oncol 2012, 38(7):630-636.

11. Staehler G, Brkovic D: The role of radical surgery for renal cell carcinoma with extension into the vena cava. J Urol 2000, 163(6):1671-1675.

12. Neves RJ, Zincke H: Surgical treatment of renal cancer with vena cava extension. Brit J Urol 1987, 59(5):390-395.

13. Sobin LH, Gospodarowicz MK, Wittekind C, International Union against Cancer: TNM classification of malignant tumours. 7th edition. Chichester, West Sussex, UK, Hoboken, NJ: Wiley-Blackwell; 2010.

14. Edge SB, American Joint Committee on Cancer: AJCC cancer staging manual. 7th edition. New YorkLondon: Springer; 2010. 
15. Oken MM, Creech RH, Tormey DC, Horton J, Davis TE, McFadden ET, Carbone PP: Toxicity and response criteria of the Eastern Cooperative Oncology Group. Am J Clin Oncol 1982, 5(6):649-655.

16. Takeda H, Nakano Y, Kashiwagi Y, Yoshino Y, Gotoh M: Downsizing a thrombus of advanced renal cell carcinoma in a presurgical setting with sorafenib. Urol Int 2012, 88(2):235-237.

17. Gettman MT, Boelter CW, Cheville JC, Zincke H, Bryant SC, Blute ML: Charlson co-morbidity index as a predictor of outcome after surgery for renal cell carcinoma with renal vein, vena cava or right atrium extension. J Urol 2003, 169(4):1282-1286

18. Haferkamp A, Bastian PJ, Jakobi H, Pritsch M, Pfitzenmaier J, Albers P, Hallscheidt P, Muller SC, Hohenfellner M: Renal cell carcinoma with tumor thrombus extension into the vena cava: prospective long-term followup. J Urol 2007, 177(5):1703-1708.

19. Martinez-Salamanca Jl, Huang WC, Millan I, Bertini R, Bianco FJ, Carballido JA, Ciancio G, Hernandez C, Herranz F, Haferkamp A, et al: Prognostic impact of the 2009 UICC/AJCC TNM staging system for renal cell carcinoma with venous extension. Eur Urol 2011, 59(1):120-127.

20. Kim HL, Zisman A, Han KR, Figlin RA, Belldegrun AS: Prognostic significance of venous thrombus in renal cell carcinoma. Are renal vein and inferior vena cava involvement different? J Urol 2004, 171(2 Pt 1):588-591.

21. Vergho DC, Loeser A, Kocot A, Spahn M, Riedmiller H: Tumor thrombus of inferior vena cava in patients with renal cell carcinoma - clinical and oncological outcome of 50 patients after surgery. BMC Res Notes 2012, 5:5.

22. Lambert EH, Pierorazio PM, Shabsigh A, Olsson CA, Benson MC, McKiernan $J M$ : Prognostic risk stratification and clinical outcomes in patients undergoing surgical treatment for renal cell carcinoma with vascular tumor thrombus. Urology 2007, 69(6):1054-1058.

23. Bex A, Van der Veldt AA, Blank C, Meijerink MR, Boven E, Haanen JB: Progression of a caval vein thrombus in two patients with primary renal cell carcinoma on pretreatment with sunitinib. Acta Oncol 2010, 49(4):520-523.

24. Karakiewicz PI, Suardi N, Jeldres C, Audet P, Ghosn P, Patard JJ, Perrotte P: Neoadjuvant sutent induction therapy may effectively down-stage renal cell carcinoma atrial thrombi. Eur Urol 2008, 53(4):845-848.

25. Harshman LC, Srinivas S, Kamaya A, Chung BI: Laparoscopic radical nephrectomy after shrinkage of a caval tumor thrombus with sunitinib. Nat Rev Urol 2009, 6(6):338-343.

26. Cost NG, Delacroix SE Jr, Sleeper JP, Smith PJ, Youssef RF, Chapin BF, Karam JA, Culp S, Abel EJ, Brugarolas J, et al: The impact of targeted molecular therapies on the level of renal cell carcinoma vena caval tumor thrombus. Eur Urol 2011, 59(6):912-918.

doi:10.1186/1471-2490-13-47

Cite this article as: Hatakeyama et al:: Prognostic benefit of surgical management in renal cell carcinoma patients with thrombus extending to the renal vein and inferior vena cava: 17-year experience at a single center. BMC Urology 2013 13:47.

\section{Submit your next manuscript to BioMed Central and take full advantage of:}

- Convenient online submission

- Thorough peer review

- No space constraints or color figure charges

- Immediate publication on acceptance

- Inclusion in PubMed, CAS, Scopus and Google Scholar

- Research which is freely available for redistribution 